\title{
Sufficiency and Duality in Nonsmooth Multiobjective Programming Problem under Generalized Univex Functions
}

\author{
Pallavi Kharbanda, ${ }^{1}$ Divya Agarwal, ${ }^{2}$ and Deepa Sinha ${ }^{3}$ \\ ${ }^{1}$ Centre for Mathematical Sciences, Banasthali University, Rajasthan 304022, India \\ ${ }^{2}$ Department of Applied Sciences and Humanities, ITM University, Gurgaon 122017, India \\ ${ }^{3}$ Department of Mathematics, South Asian University, New Delhi 110021, India
}

Correspondence should be addressed to Pallavi Kharbanda; pallavimath@yahoo.co.in

Received 18 February 2014; Accepted 25 March 2014; Published 22 May 2014

Academic Editors: P.-y. Nie and S. Utyuzhnikov

Copyright (C) 2014 Pallavi Kharbanda et al. This is an open access article distributed under the Creative Commons Attribution License, which permits unrestricted use, distribution, and reproduction in any medium, provided the original work is properly cited.

\begin{abstract}
We consider a nonsmooth multiobjective programming problem where the functions involved are nondifferentiable. The class of univex functions is generalized to a far wider class of $(\varphi, \alpha, \rho, \sigma)-d_{I}-V$-type I univex functions. Then, through various nontrivial examples, we illustrate that the class introduced is new and extends several known classes existing in the literature. Based upon these generalized functions, Karush-Kuhn-Tucker type sufficient optimality conditions are established. Further, we derive weak, strong, converse, and strict converse duality theorems for Mond-Weir type multiobjective dual program.
\end{abstract}

\section{Introduction}

Generalizations of convexity related to optimality conditions and duality for nonlinear single objective or multiobjective optimization problems have been of much interest in the recent past and thus explored the extent of optimality conditions and duality applicability in mathematical programming problems. Consequently, various generalizations of convex functions have been introduced in the literature (see Hanson [1], Vial [2], Hanson and Mond [3], Jeyakumar and Mond [4], Hanson et al. [5], Liang et al. [6], and Gulati et al. [7]).

Nonsmooth optimization provides analytical tools for studying optimization problems involving functions that are not differentiable in the usual sense. Several nonlinear analysis problems arise from areas of optimization theory, game theory, differential equations, mathematical physics, convex analysis, and nonlinear functional analysis. For a nondifferentiable multiobjective programming problem, there exists a generalization of invexity to locally Lipschitz functions with gradients replaced by the Clarke generalized subgradient. Instead of Clarke generalized subgradient, Ye [8] used the concept of directional derivative to define the class of $d$ invex functions. Also, he derived necessary and sufficient optimality conditions taking functions $f^{\prime}\left(x_{o} ; y\right)$ and $g_{J}^{\prime}\left(x_{o} ; y\right)$ to be convex. However, Antczak [9] considered the directional derivatives of objective and constraint functions to be preinvex and derived duality results for Wolfe type, MondWeir type, and mixed type dual programs. Mishra and Noor [10] extended the class of functions to $d$ - $V$-type I functions and obtained sufficient optimality and duality results for Mond-Weir type multiobjective dual program. Nahak and Mohapatra [11] obtained duality results for multiobjective programming problem under $(d-\rho-\eta-\theta)$ invexity assumptions. Slimani and Radjef [12] introduced a far wider class of nondifferentiable functions called $d_{I}-V$-type I functions in which each component is directionally differentiable in its own direction instead of the same direction and established sufficient optimality and duality results.

On the other hand, Bector et al. [13] generalized the notion of convexity to univex functions. Rueda et al. [14] obtained optimality and duality results for several mathematical programs by combining the concepts of type I and univex functions. Mishra [15] obtained optimality results and saddle point results for multiobjective programs under generalized 
type I univex functions. Generalizing the functions, Mishra et al. [16] obtained duality results for a nondifferentiable multiobjective programming problem under generalized $d$ univexity. As an extension, Ahmad [17] introduced a new class of $d_{I}$ - $V$-type I univex functions which was generalized to a class of $\left(d_{I}-\rho-\sigma\right)-V$-type I univex functions by Kharbanda et al. [18].

In this paper, we introduce a new generalized class of $(\varphi, \alpha, \rho, \sigma)-d_{I}-V$-type I univex functions which generalizes the class of functions introduced by Kharbanda et al. [18], Ahmad [17], Slimani and Radjef [12], Mishra and Noor [10], Mishra et al. [16], Antczak [9], Suneja and Srivastava [19], and Ye [8]. Further, we establish weak, strong, converse, and strict converse duality results for Mond-Weir type multiobjective dual program.

\section{Preliminaries and Definitions}

The following convention of vectors in $R^{n}$ will be followed throughout this paper: $x \geqq y \Leftrightarrow x_{i} \geqq y_{i}, i=1,2, \ldots, n$; $x \geq y \Leftrightarrow x \geqq y, x \neq y ; x>y \Leftrightarrow x_{i}>y_{i}, i=1,2, \ldots, n$. Let $D$ be a nonempty subset of $R^{n}, \eta: D \times D \rightarrow R^{n}$, and let $x_{o}$ be an arbitrary point of $D$ and $h: D \rightarrow R, f: D \rightarrow R^{m}$, $\phi: R \rightarrow R, b: D \times D \rightarrow R_{+}$. Also, we denote $R_{\geq}^{m}=\{y: y \in$ $R^{m}$ and $\left.y \geq 0\right\}, R_{\geqq(>)}^{k}=\left\{y: y \in R^{k}\right.$ and $\left.y \geqq 0(y>0)\right\}$ and $i=\overline{1, m}=\{1,2, \ldots, m\}, j=\overline{1, k}=\{1,2, \ldots, k\}$.

Definition 1 (Weir and Mond [20] and Weir and Jeyakumar [21]). The function $h$ is called preinvex on $D$ if, for all $x, x_{o} \in$ $D$, there exists a vector function $\eta$ such that $\forall \lambda \in[0,1], x_{0}+$ $\lambda \eta\left(x, x_{o}\right) \in D$, one has

$$
\lambda h(x)+(1-\lambda) h\left(x_{o}\right) \geqq h\left(x_{o}+\lambda \eta\left(x, x_{o}\right)\right) .
$$

Definition 2 (Mititelu [22]). The set $D$ is said to be invex at $x_{o}$ with respect to $\eta$, if, for each $x \in D$,

$$
x_{o}+\lambda \eta\left(x, x_{o}\right) \in D, \quad \forall \lambda \in[0,1] .
$$

$D$ is said to be an invex set with respect to $\eta$, if $D$ is invex at each $x_{o} \in D$ with respect to same $\eta$.

Definition 3 (Antczak [9]). Let $D \subseteq R^{n}$ be an invex set. An $m$-dimensional vector-valued function $\psi: D \rightarrow R^{m}$ is said to be preinvex with respect to $\eta$, if each of its components is preinvex on $D$ with respect to the same function $\eta$.

Definition 4 (Clarke [23]). The function $h$ is said to be locally Lipschitz at $x_{o} \in D$, if there exist a neighbourhood $v\left(x_{o}\right)$ of $x_{o}$ and a constant $k>0$ such that

$$
|h(y)-h(x)| \leqq k\|y-x\|, \quad \forall x, y \in v\left(x_{o}\right),
$$

where $\|\cdot\|$ denotes the Euclidean norm. Also, one says that $h$ is locally Lipschitz on $D$ if it is locally Lipschitz at every point of $D$.

Definition 5 (Bector et al. [13]). A differentiable function $h$ is said to be univex at $x_{o}$ with respect to $\phi, \eta, b$ if, $\forall x \in D$, one has

$$
b\left(x, x_{o}\right) \phi\left(h(x)-h\left(x_{o}\right)\right) \geqq\left[\nabla h\left(x_{o}\right)\right]^{T} \eta\left(x, x_{o}\right) .
$$

Definition 6 (Mishra et al. [16]). Let $D \subseteq R^{n}$ be a nonempty open set. The function $f$ is called $d$-univex at $x_{o} \in D$ with respect to $\phi, \eta, b$ if it is directionally differentiable at $x_{o}$ such that, for any $x \in D$,

$$
\begin{array}{r}
b\left(x, x_{o}\right) \phi\left(f_{i}(x)-f_{i}\left(x_{o}\right)\right) \geqq f_{i}^{\prime}\left(x_{o} ; \eta\left(x, x_{o}\right)\right), \\
\forall i=\overline{1, m},
\end{array}
$$

where $f_{i}^{\prime}\left(x_{o} ; \eta\left(x, x_{o}\right)\right)$ denotes the directional derivative of $f_{i}$ at $x_{o}$ in the direction $\eta\left(x, x_{o}\right)$ :

$$
\begin{aligned}
& f_{i}^{\prime}\left(x_{o} ; \eta\left(x, x_{o}\right)\right) \\
& \quad=\lim _{\lambda \rightarrow 0^{+}} \frac{f_{i}\left(x_{o}+\lambda \eta\left(x, x_{o}\right)\right)-f_{i}\left(x_{o}\right)}{\lambda} .
\end{aligned}
$$

If the above inequalities are satisfied at any point $x_{o} \in D$, then $f$ is said to be $d$-univex on $D$ with respect to $\eta$.

Definition 7 (Slimani and Radjef [12]). The function $h$ is said to be semidirectionally differentiable at $x_{0} \in D$ in the direction $\eta\left(x, x_{o}\right)$ if its directional derivative $h^{\prime}\left(x_{o} ; \eta\left(x, x_{o}\right)\right)$ exists finite for all $x \in D$.

Definition 8 (Slimani and Radjef [12]). Let $D \subseteq R^{n}$ be a nonempty open set. The function $f$ is called $d_{I}$-invex at $x_{o} \in$ $D$ with respect to $\left(\eta_{i}\right)_{i=\overline{1, m}}$, if, for any $x \in D$,

$$
f_{i}(x)-f_{i}\left(x_{o}\right) \geqq f_{i}^{\prime}\left(x_{o} ; \eta_{i}\left(x, x_{o}\right)\right), \quad \forall i=\overline{1, m},
$$

where $f_{i}$ is semidirectionally differentiable at $x_{o}$ in direction $\eta_{i}: D \times D \rightarrow R^{n}$, for $i=\overline{1, m}$.

We consider the following nonlinear multiobjective programming problem:

$(\mathrm{MP})$

$$
\begin{array}{ll}
\text { Minimize } & f(x)=\left(f_{1}(x), f_{2}(x), \ldots, f_{m}(x)\right) \\
\text { subject to } & g(x) \leqq 0
\end{array}
$$

where $x \in D$ and the functions $f: D \rightarrow R^{m}, g: D \rightarrow R^{k}$, and $D$ is a nonempty open subset of $R^{n}$. Let $X=\{x \in D$ : $g(x) \leqq 0\}$ be the set of feasible solutions of (MP). For $x_{o} \in D$, if we denote

$$
\begin{aligned}
& J\left(x_{o}\right)=\left\{j \in\{1,2, \ldots, k\}: g_{j}\left(x_{o}\right)=0\right\}, \\
& \widetilde{J}\left(x_{o}\right)=\left\{j \in\{1,2, \ldots, k\}: g_{j}\left(x_{o}\right)<0\right\}, \\
& \bar{J}\left(x_{o}\right)=\left\{j \in\{1,2, \ldots, k\}: g_{j}\left(x_{o}\right)>0\right\},
\end{aligned}
$$

then

$$
J\left(x_{o}\right) \cup \widetilde{J}\left(x_{o}\right) \cup \bar{J}\left(x_{o}\right)=\{1,2, \ldots, k\}
$$


Now we define a new class of $(\varphi, \alpha, \rho, \sigma)-d_{I}-V$-type I univex functions where $\varphi: D \times D \times R \rightarrow R$ is a functional which for any $x, x_{o} \in D$ satisfies the following properties:

(i) $\varphi\left(x, x_{o} ; a_{1}+a_{2}\right) \leqq \varphi\left(x, x_{o} ; a_{1}\right)+\varphi\left(x, x_{o} ; a_{2}\right)$, for all $a_{1}, a_{2} \in R$, (subadditive in third argument);

(ii) $\varphi\left(x, x_{o} ; \alpha a\right)=\alpha \varphi\left(x, x_{o} ; a\right)$, for all $\alpha \in R, \alpha \geqq 0$ and for all $a \in R$, (positive homogeneous in third argument);

(iii) $\varphi\left(x, x_{o} ; a\right)>0$, for all $a>0$.

Let the functions $f: D \rightarrow R^{m}$ and $g: D \rightarrow R^{k}$ where $f_{i}$ and $g_{j}$ are semidirectionally differentiable functions in the directions $\eta_{i}: X \times D \rightarrow R^{n}$ and $\theta_{j}: X \times D \rightarrow R^{n}$ for $i=\overline{1, m}$ and $j=\overline{1, k}$. Also, let $\alpha, \tilde{\alpha}$ be the vectors in $R^{m+k}$ whose components are the functions $\alpha_{i}^{1}, \alpha_{j}^{2}: X \times D \rightarrow R_{+}$। $\{0\}, \tilde{\alpha}_{i}^{1}, \widetilde{\alpha}_{j}^{2}: X \times D \rightarrow R_{+} \backslash\{0\}$, respectively, for $i=\overline{1, m}$, $j=\overline{1, k}$, while $\rho \in R^{m+k}$ and $\tilde{\rho} \in R^{2}$ whose components are in $R$ and $\sigma: X \times D \rightarrow R^{n} ; b_{o}$ and $b_{1}$ are nonnegative functions defined on $X \times D, \phi_{o}: R \rightarrow R$, and $\phi_{1}: R \rightarrow R$.

Definition 9. $(f, g)$ is said to be $(\varphi, \alpha, \rho, \sigma)-d_{I}-V$-type I univex at $x_{o} \in D$ with respect to $\left(\eta_{i}\right)_{i=\overline{1, m}}$ and $\left(\theta_{j}\right)_{j=\overline{1, k}}$ if for all $x \in X$

$$
\begin{aligned}
& b_{o}\left(x, x_{o}\right) \phi_{o}\left(f_{i}(x)-f_{i}\left(x_{o}\right)\right) \\
& \geqq \varphi\left(x, x_{o} ; \alpha_{i}^{1}\left(x, x_{o}\right) f_{i}^{\prime}\left(x_{o} ; \eta_{i}\left(x, x_{o}\right)\right)\right) \\
& \quad+\rho_{i}^{1}\left\|\sigma\left(x, x_{o}\right)\right\|^{2}, \quad \forall i=\overline{1, m}, \\
& -b_{1}\left(x, x_{o}\right) \phi_{1}\left(g_{j}\left(x_{o}\right)\right) \\
& \geqq \varphi\left(x, x_{o} ; \alpha_{j}^{2}\left(x, x_{o}\right) g_{j}^{\prime}\left(x_{o} ; \theta_{j}\left(x, x_{o}\right)\right)\right) \\
& \quad+\rho_{j}^{2}\left\|\sigma\left(x, x_{o}\right)\right\|^{2}, \quad \forall j=\overline{1, k} .
\end{aligned}
$$

If the inequalities in $f$ are strict (whenever $x \neq x_{0}$ ), then $(f, g)$ is said to be semistrictly $(\varphi, \alpha, \rho, \sigma)-d_{I^{-}} V$-type I univex at $x_{o}$ with respect to $\left(\eta_{i}\right)_{i=\overline{1, m}}$ and $\left(\theta_{j}\right)_{j=\overline{1, k}}$.

Remark 10. (i) If in the above definition, $\varphi\left(x, x_{o} ; \alpha_{i}^{1}\left(x, x_{o}\right)\right.$ $\left.f_{i}^{\prime}\left(x_{o} ; \eta_{i}\left(x, x_{o}\right)\right)\right)=\alpha_{i}^{1}\left(x, x_{o}\right) f_{i}^{\prime}\left(x_{o} ; \eta_{i}\left(x, x_{o}\right)\right), \varphi\left(x, x_{o} ; \alpha_{j}^{2}(x\right.$, $\left.\left.x_{o}\right) g_{j}^{\prime}\left(x_{o} ; \theta_{j}\left(x, x_{o}\right)\right)\right)=\alpha_{j}^{2}\left(x, x_{o}\right) g_{j}^{\prime}\left(x_{o} ; \theta_{j}\left(x, x_{o}\right)\right)$, for all $i=$ $\overline{1, m}$ and $j=\overline{1, k}$, then we obtain the definition of $\left(d_{I^{-}}\right.$ $\rho-\sigma)-V$-type I univex function given by Kharbanda et al. [18]. Also, if in addition, we take $\rho_{i}^{1}, \rho_{j}^{2}=0$, then the above definition reduces to definition of $d_{I}-V$-type I univex function introduced by Ahmad [17].

(ii) If $\varphi$ is same as in (i) and $\rho_{i}^{1}, \rho_{j}^{2}=0, \alpha_{i}^{1}\left(x, x_{o}\right)=$ $\alpha_{j}^{2}\left(x, x_{o}\right)=1$, for all $i=\overline{1, m}$ and $j=\overline{1, k}$ and $b_{o}\left(x, x_{o}\right)=$ $b_{1}\left(x, x_{o}\right)=1, \phi_{o}(t)=t$, and $\phi_{1}(t)=t$, then the above definition becomes definition of $d_{I}$-V-type I function introduced by Slimani and Radjef [12].

(iii) If $f$ and $g$ are differentiable functions and $\varphi\left(x, x_{o}\right.$; $\left.\alpha_{i}^{1}\left(x, x_{o}\right) f_{i}^{\prime}\left(x_{o} ; \eta_{i}\left(x, x_{o}\right)\right)\right)=\alpha_{i}^{1}\left(x, x_{o}\right)\left[\nabla f_{i}\left(x_{o}\right)\right]^{T} \eta\left(x, x_{o}\right)$, $\varphi\left(x, x_{o} ; \alpha_{j}^{2}\left(x, x_{o}\right) g_{j}^{\prime}\left(x_{o} ; \theta_{j}\left(x, x_{o}\right)\right)\right)=\alpha_{j}^{2}\left(x, x_{o}\right)\left[\nabla g_{j}\left(x_{o}\right)\right]^{T}$ $\eta\left(x, x_{o}\right)$, and $\rho_{i}^{1}, \rho_{j}^{2}=0, i=\overline{1, m}$ and $j=\overline{1, k}$ and $b_{o}\left(x, x_{o}\right)=$ $b_{1}\left(x, x_{o}\right)=1, \phi_{o}(t)=t, \phi_{1}(t)=t$, then above definition reduces to $V$-type I functions given by Hanson et al. [5]. Also, if, in addition, we take $\alpha_{i}^{1}\left(x, x_{o}\right)=\alpha_{j}^{2}\left(x, x_{o}\right)=1$, then we get the definition of type I function defined by Hanson and Mond [3].

(iv) If, in the above definition, $\varphi\left(x, x_{o} ; \alpha_{i}^{1}\left(x, x_{o}\right) f_{i}^{\prime}\left(x_{o} ; \eta_{i}\right.\right.$ $\left.\left.\left(x, x_{o}\right)\right)\right)=\alpha_{i}^{1}\left(x, x_{o}\right) f_{i}^{\prime}\left(x_{o} ; \eta\left(x, x_{o}\right)\right), \varphi\left(x, x_{o} ; \alpha_{j}^{2}\left(x, x_{o}\right) g_{j}^{\prime}\left(x_{o} ;\right.\right.$ $\left.\left.\theta_{j}\left(x, x_{o}\right)\right)\right)=\alpha_{j}^{2}\left(x, x_{o}\right) g_{j}^{\prime}\left(x_{o} ; \eta\left(x, x_{o}\right)\right)$, and $\rho_{i}^{1}, \rho_{j}^{2}=0, i=$ $\overline{1, m}$, and $j=\overline{1, k}$ and $b_{o}\left(x, x_{o}\right)=b_{1}\left(x, x_{o}\right)=1, \phi_{o}(t)=t$, and $\phi_{1}(t)=t$, then we obtain the definition of $d$-type I function introduced by Suneja and Srivastava [19].

Definition 11. $(f, g)$ is said to be quasi $(\varphi, \tilde{\alpha}, \tilde{\rho}, \sigma)-d_{I}-V$-type I univex at $x_{o} \in D$ with respect to $\left(\eta_{i}\right)_{i=\overline{1, m}}$ and $\left(\theta_{j}\right)_{j=\overline{1, k}}$, if for some vectors $\mu \in R_{\geqq}^{m}, \lambda \in R_{\geqq}^{k}$ and for all $x \in X$

$$
\begin{aligned}
b_{o}\left(x, x_{o}\right) \phi_{o}\left(\sum_{i=1}^{m} \mu_{i} \tilde{\alpha}_{i}^{1}\left(x, x_{o}\right)\left(f_{i}(x)-f_{i}\left(x_{o}\right)\right)\right) \leqq 0 \\
\Longrightarrow \varphi\left(x, x_{o} ; \sum_{i=1}^{m} \mu_{i} f_{i}^{\prime}\left(x_{o} ; \eta_{i}\left(x, x_{o}\right)\right)\right) \\
\leqq-\widetilde{\rho}^{1}\left\|\sigma\left(x, x_{o}\right)\right\|^{2}, \\
b_{1}\left(x, x_{o}\right) \phi_{1}\left(\sum_{j=1}^{k} \lambda_{j} \tilde{\alpha}_{j}^{2}\left(x, x_{o}\right) g_{j}\left(x_{o}\right)\right) \geqq 0 \\
\Longrightarrow \varphi\left(x, x_{o} ; \sum_{j=1}^{k} \lambda_{j} g_{j}^{\prime}\left(x_{o} ; \theta_{j}\left(x, x_{o}\right)\right)\right) \\
\leqq-\widetilde{\rho}^{2}\left\|\sigma\left(x, x_{o}\right)\right\|^{2} .
\end{aligned}
$$

If the second (implied) inequality in $f$ is strict $\left(x \neq x_{o}\right)$, then $(f, g)$ is said to be semistrictly quasi $(\varphi, \tilde{\alpha}, \tilde{\rho}, \sigma)-d_{I}-V$ type I univex at $x_{o}$ with respect to $\left(\eta_{i}\right)_{i=\overline{1, m}}$ and $\left(\theta_{j}\right)_{j=1, k}$.

Definition 12. $(f, g)$ is said to be pseudo $(\varphi, \tilde{\alpha}, \tilde{\rho}, \sigma)-d_{I}-V$ type I univex at $x_{o} \in D$ with respect to $\left(\eta_{i}\right)_{i=\overline{1, m}}$ and $\left(\theta_{j}\right)_{j=\overline{1, k}}$, if for some vectors $\mu \in R_{\geqq}^{m}, \lambda \in R_{\geqq}^{k}$ and for all $x \in X$

$$
\begin{aligned}
& \varphi\left(x, x_{o} ; \sum_{i=1}^{m} \mu_{i} f_{i}^{\prime}\left(x_{o} ; \eta_{i}\left(x, x_{o}\right)\right)\right) \\
& \geqq-\tilde{\rho}^{1}\left\|\sigma\left(x, x_{o}\right)\right\|^{2} \\
& \Longrightarrow b_{o}\left(x, x_{o}\right) \phi_{o}\left(\sum_{i=1}^{m} \mu_{i} \widetilde{\alpha}_{i}^{1}\left(x, x_{o}\right)\left(f_{i}(x)-f_{i}\left(x_{o}\right)\right)\right)
\end{aligned}
$$

$\geqq 0$, 


$$
\begin{aligned}
\varphi(x, & \left.x_{o} ; \sum_{j=1}^{k} \lambda_{j} g_{j}^{\prime}\left(x_{o} ; \theta_{j}\left(x, x_{o}\right)\right)\right) \\
& \geqq-\widetilde{\rho}^{2}\left\|\sigma\left(x, x_{o}\right)\right\|^{2} \\
& \Longrightarrow b_{1}\left(x, x_{o}\right) \phi_{1}\left(\sum_{j=1}^{k} \lambda_{j} \widetilde{\alpha}_{j}^{2}\left(x, x_{o}\right) g_{j}\left(x_{o}\right)\right) \leqq 0 .
\end{aligned}
$$

If the second (implied) inequality in $f$ (resp., $g$ ) is strict $\left(x \neq x_{o}\right)$, then $(f, g)$ is said to be semistrictly pseudo $(\varphi, \widetilde{\alpha}, \widetilde{\rho}, \sigma)-d_{I}-V$-type I univex in $f$ (resp., $\left.g\right)$ and if the second (implied) inequalities in $f$ and $g$ are both strict, then $(f, g)$ is said to be strictly pseudo $(\varphi, \widetilde{\alpha}, \widetilde{\rho}, \sigma)-d_{I}-V$-type I univex at $x_{o}$ with respect to $\left(\eta_{i}\right)_{i=\overline{1, m}}$ and $\left(\theta_{j}\right)_{j=\overline{1, k}}$.

Definition 13. $(f, g)$ is said to be quasi-pseudo $(\varphi, \tilde{\alpha}, \tilde{\rho}, \sigma)$ $d_{I}$-V-type I univex at $x_{o} \in D$ with respect to $\left(\eta_{i}\right)_{i=\overline{1, m}}$ and $\left(\theta_{j}\right)_{j=\overline{1, k}}$, if for some vectors $\mu \in R_{\geqq}^{m}, \lambda \in R_{\geqq}^{k}$ and for all $x \in X$

$$
\begin{gathered}
b_{o}\left(x, x_{o}\right) \phi_{o}\left(\sum_{i=1}^{m} \mu_{i} \widetilde{\alpha}_{i}^{1}\left(x, x_{o}\right)\left(f_{i}(x)-f_{i}\left(x_{o}\right)\right)\right) \leqq 0 \\
\Longrightarrow \varphi\left(x, x_{o} ; \sum_{i=1}^{m} \mu_{i} f_{i}^{\prime}\left(x_{o} ; \eta_{i}\left(x, x_{o}\right)\right)\right) \\
\leqq-\widetilde{\rho}^{1}\left\|\sigma\left(x, x_{o}\right)\right\|^{2}, \\
\varphi\left(x, x_{o} ; \sum_{j=1}^{k} \lambda_{j} g_{j}^{\prime}\left(x_{o} ; \theta_{j}\left(x, x_{o}\right)\right)\right) \\
\geqq-\widetilde{\rho}^{2}\left\|\sigma\left(x, x_{o}\right)\right\|^{2} \\
\Longrightarrow b_{1}\left(x, x_{o}\right) \phi_{1}\left(\sum_{j=1}^{k} \lambda_{j} \widetilde{\alpha}_{j}^{2}\left(x, x_{o}\right) g_{j}\left(x_{o}\right)\right) \leqq 0 .
\end{gathered}
$$

If the second (implied) inequality in $g$ is strict $\left(x \neq x_{0}\right)$, then $(f, g)$ is said to be quasistrictly pseudo $(\varphi, \widetilde{\alpha}, \widetilde{\rho}, \sigma)-d_{I}-V$ type I univex at $x_{o}$ with respect to $\left(\eta_{i}\right)_{i=1, m}$ and $\left(\theta_{j}\right)_{j=\overline{1, k}}$.

Definition 14. $(f, g)$ is said to be pseudo-quasi $(\varphi, \widetilde{\alpha}, \widetilde{\rho}, \sigma)$ $d_{I}$-V-type I univex at $x_{o} \in D$ with respect to $\left(\eta_{i}\right)_{i=1, m}$ and $\left(\theta_{j}\right)_{j=\overline{1, k}}$, if for some vectors $\mu \in R_{\geqq}^{m}, \lambda \in R_{\geqq}^{k}$ and for all $x \in X$

$$
\begin{aligned}
\varphi(x, & \left.x_{o} ; \sum_{i=1}^{m} \mu_{i} f_{i}^{\prime}\left(x_{o} ; \eta_{i}\left(x, x_{o}\right)\right)\right) \\
& \geqq-\tilde{\rho}^{1}\left\|\sigma\left(x, x_{o}\right)\right\|^{2} \\
& \Longrightarrow b_{o}\left(x, x_{o}\right) \phi_{o}\left(\sum_{i=1}^{m} \mu_{i} \tilde{\alpha}_{i}^{1}\left(x, x_{o}\right)\left(f_{i}(x)-f_{i}\left(x_{o}\right)\right)\right)
\end{aligned}
$$

$\geqq 0$,

$$
\begin{aligned}
& b_{1}\left(x, x_{o}\right) \phi_{1}\left(\sum_{j=1}^{k} \lambda_{j} \tilde{\alpha}_{j}^{2}\left(x, x_{o}\right) g_{j}\left(x_{o}\right)\right) \geqq 0 \\
& \Longrightarrow \varphi\left(x, x_{o} ; \sum_{j=1}^{k} \lambda_{j} g_{j}^{\prime}\left(x_{o} ; \theta_{j}\left(x, x_{o}\right)\right)\right) \\
& \leqq-\tilde{\rho}^{2}\left\|\sigma\left(x, x_{o}\right)\right\|^{2} .
\end{aligned}
$$

If the second (implied) inequality in $f$ is strict $\left(x \neq x_{o}\right)$, then $(f, g)$ is said to be strictly pseudo-quasi $(\varphi, \tilde{\alpha}, \tilde{\rho}, \sigma)-d_{I^{-}}$ $V$-type I univex at $x_{o}$ with respect to $\left(\eta_{i}\right)_{i=\overline{1, m}}$ and $\left(\theta_{j}\right)_{j=\overline{1, k}}$.

\section{Illustration}

In this section, we give some nontrivial examples which illustrate that the class of functions introduced in this paper is nonempty.

Example 15. Let $f: R^{2} \rightarrow R$ and $g: R^{2} \rightarrow R$ be defined by

$$
\begin{gathered}
f\left(x_{1}, x_{2}\right)= \begin{cases}x_{1}^{2}+x_{2}^{2}+\left|x_{1}\right|+4 & \text { if } x_{1} \neq 0, x_{2} \neq 0 \\
4 & \text { if } x_{1}=0, x_{2}=0 \\
8+x_{1}^{2}+x_{2}^{2} & \text { else, }\end{cases} \\
g\left(x_{1}, x_{2}\right)= \begin{cases}-2-4 x_{1}^{2}-6 x_{2}^{2} & \text { if } x_{1} \neq 0, x_{2} \neq 0 \\
0 & \text { else. }\end{cases}
\end{gathered}
$$

Let $\eta(x, y)=\left(1+x_{1}^{2}, 2+y_{2}^{2}\right), \theta(x, y)=\left(0, x_{2}\right), \varphi(x, y ; a)=$ $(|a| / 2)\left(1+x_{1}^{2} y_{2}^{2}\right), \sigma(x, y)=\left(x_{1}+y_{1}, 1+y_{2}\right)$, and $\alpha^{1}(x, y)=$ $8, \alpha^{2}(x, y)=4$ where $x=\left(x_{1}, x_{2}\right), y=\left(y_{1}, y_{2}\right)$.

Also, let $b_{o}(x, y)=1 / 2, b_{1}(x, y)=4, \phi_{o}(t)=4 t, \phi_{1}(t)=$ $4 t, \rho^{1}=-4$, and $\rho^{2}=-2$.

The set $X$ of feasible solutions of problem is nonempty. Clearly, $f$ and $g$ are semidirectionally differentiable at $x_{o}=$ $(0,0)$ with $f^{\prime}\left(x_{o} ; \eta\left(x, x_{o}\right)\right)=1+x_{1}^{2}$ and $g^{\prime}\left(x_{o} ; \theta\left(x, x_{o}\right)\right)=0$.

It is easy to see that for all $x \in X$

$$
\begin{aligned}
& b_{o}\left(x, x_{o}\right) \phi_{o}\left(f(x)-f\left(x_{o}\right)\right) \\
& \geqq \varphi\left(x, x_{o} ; \alpha^{1}\left(x, x_{o}\right) f^{\prime}\left(x_{o} ; \eta\left(x, x_{o}\right)\right)\right) \\
& \quad+\rho^{1}\left\|\sigma\left(x, x_{o}\right)\right\|^{2}, \\
& -b_{1}\left(x, x_{o}\right) \phi_{1}\left(g\left(x_{o}\right)\right) \\
& \geqq \varphi\left(x, x_{o} ; \alpha^{2}\left(x, x_{o}\right) g^{\prime}\left(x_{o} ; \theta\left(x, x_{o}\right)\right)\right) \\
& \quad+\rho^{2}\left\|\sigma\left(x, x_{o}\right)\right\|^{2} .
\end{aligned}
$$

Therefore $(f, g)$ is $(\varphi, \alpha, \rho, \sigma)-d_{I^{-}} V$-type I function at $x_{o}$.

However, if we take $x=(1,1)$, then

(i) $b_{o}(x, x) \phi_{o}\left(f(x)-f\left(x_{o}\right)\right)$

$$
<\alpha^{1}\left(x, x_{o}\right) f^{\prime}\left(x_{o} ; \eta\left(x, x_{o}\right)\right)+\rho^{1}\left\|\sigma\left(x, x_{o}\right)\right\|^{2},
$$

$b_{o}(x, x) \phi_{o}\left(f(x)-f\left(x_{o}\right)\right)<\alpha^{1}\left(x, x_{o}\right) f^{\prime}\left(x_{o} ; \eta\left(x, x_{o}\right)\right)$. 
Thus $(f, g)$ is neither $\left(d_{I}-\rho-\sigma\right)-V$-type I univex function given by Kharbanda et al. [18] nor $d_{I}$-V-type I univex function at $x_{o}$ as given by Ahmad [17].

Hence the above example clearly illustrates that the class of $(\varphi, \alpha, \rho, \sigma)-d_{I}-V$-type I univex functions is more generalized than the class of $\left(d_{I}-\rho-\sigma\right)-V$-type I univex functions and the class of $d_{I}-V$-type I univex functions.

Next we show that $(f, g)$ is pseudo-quasi $(\varphi, \widetilde{\alpha}, \tilde{\rho}, \sigma)-d_{I^{-}}$ $V$-type I univex function but not $(\varphi, \alpha, \rho, \sigma)-d_{I}-V$-type I univex function.

Example 16. Let $f: R^{2} \rightarrow R^{3}$ and $g: R^{2} \rightarrow R^{2}$ be defined by

$$
\begin{aligned}
& f_{1}\left(x_{1}, x_{2}\right)= \begin{cases}4 x_{1}^{2}-2 x_{2}+4 x_{2}^{2} & \text { if } x_{1} \neq 0, x_{2} \neq 0 \\
9+9 x_{2}^{2} & \text { if } x_{1}=0, x_{2} \neq 0 \\
0 & \text { else, }\end{cases} \\
& f_{2}\left(x_{1}, x_{2}\right)= \begin{cases}2 x_{2}^{2} x_{1}^{2}+6+x_{2} & \text { if } x_{1} \neq 0, x_{2} \neq 0 \\
0 & \text { else, }\end{cases} \\
& f_{3}\left(x_{1}, x_{2}\right)= \begin{cases}1+6 x_{1}^{2} & \text { if } x_{1} \neq 0, x_{2}=0 \\
2+3 x_{2}^{2} & \text { if } x_{1}=0, x_{2} \neq 0 \\
0 & \text { else, }\end{cases} \\
& g_{1}\left(x_{1}, x_{2}\right)= \begin{cases}0 & \text { if } x_{1}=0 \text { or } x_{2}=0 \\
-1+x_{1}^{2} & \text { else, }\end{cases} \\
& g_{2}\left(x_{1}, x_{2}\right)= \begin{cases}2-x_{1}^{2} & \text { if } x_{1} \neq 0, x_{2}=0 \\
2-6 x_{2}^{2} & \text { if } x_{1}=0, x_{2} \neq 0 \\
-1 & \text { else. }\end{cases}
\end{aligned}
$$

Let $\eta_{1}(x, y)=\left(1+x_{1}^{2}+y_{1}^{2}, 1+x_{2}^{2}\right), \eta_{2}(x, y)=\left(0, x_{1}+x_{2}\right)$, $\eta_{3}(x, y)=\left(4+x_{1}^{4}, 2+x_{2}^{2}+y_{2}^{2}\right), \theta_{1}(x, y)=\left(1+x_{1}, 0\right)$, $\theta_{2}(x, y)=\left(2+x_{1}^{4}, 2+2 x_{2}^{2}\right), \varphi(x, y ; a)=|a|\left(x_{1}^{2}+y_{1}^{2}\right)$, $b_{o}(x, y)=4, b_{1}(x, y)=4, \phi_{o}(t)=t / 2, \phi_{1}(t)=4 t$, and $\sigma(x, y)=\left(x_{1}+y_{1}, x_{2}+y_{2}\right)$.

Also, let $\alpha_{1}^{1}(x, y)=2, \alpha_{2}^{1}(x, y)=1, \alpha_{3}^{1}(x, y)=1$, $\alpha_{1}^{2}(x, y)=1, \alpha_{2}^{2}(x, y)=3, \tilde{\rho}^{1}=1, \widetilde{\rho}^{2}=-4, \rho_{1}^{1}=4, \rho_{2}^{1}=1$, $\rho_{3}^{1}=-6, \rho_{1}^{2}=-4, \rho_{2}^{2}=-6, \mu_{1}=\mu_{2}=1 / 2, \mu_{3}=0$, and $\lambda_{1}=1, \lambda_{2}=0$ where $x=\left(x_{1}, x_{2}\right), y=\left(y_{1}, y_{2}\right)$, $\tilde{\alpha}_{i}^{1}(x, y)=1 / \alpha_{i}^{1}(x, y)$, and $\tilde{\alpha}_{j}^{2}(x, y)=1 / \alpha_{j}^{2}(x, y), i=1,2,3$, $j=1,2$.

The set $X$ of feasible solutions of problem is nonempty. Clearly, $f_{1}, f_{2}, f_{3}$ and $g_{1}, g_{2}$ are semidirectionally differentiable at $x_{0}=(0,0)$ with $f_{1}^{\prime}\left(x_{o} ; \eta_{1}\left(x, x_{0}\right)\right)=$ $-2\left(1+x_{2}^{2}\right), f_{2}^{\prime}\left(x_{o} ; \eta_{2}\left(x, x_{o}\right)\right)=0, f_{3}^{\prime}\left(x_{o} ; \eta_{3}\left(x, x_{o}\right)\right)=0$, $g_{1}^{\prime}\left(x_{o} ; \theta_{1}\left(x, x_{o}\right)\right)=0$, and $g_{2}^{\prime}\left(x_{o} ; \theta_{2}\left(x, x_{o}\right)\right)=0$.

It is easy to see that for all $x \in X$

$$
\begin{aligned}
& \varphi\left(x, x_{o} ; \sum_{i=1}^{3} \mu_{i} f_{i}^{\prime}\left(x_{o} ; \eta_{i}\left(x, x_{o}\right)\right)\right)+\widetilde{\rho}^{1}\left\|\sigma\left(x, x_{o}\right)\right\|^{2} \\
& \quad=x_{2}^{2} x_{1}^{2}+x_{2}^{2}+2 x_{1}^{2} \geqq 0
\end{aligned}
$$

$$
\Longrightarrow b_{o}\left(x, x_{o}\right) \phi_{o}\left(\sum_{i=1}^{3} \mu_{i} \widetilde{\alpha}_{i}^{1}\left(x, x_{o}\right)\left(f_{i}(x)-f_{i}\left(x_{o}\right)\right)\right)
$$

$\geqq 0$,

$$
\begin{gathered}
b_{1}\left(x, x_{o}\right) \phi_{1}\left(\sum_{j=1}^{2} \lambda_{j} \widetilde{\alpha}_{j}^{2}\left(x, x_{o}\right) g_{j}\left(x_{o}\right)\right)=0 \geqq 0 \\
\Longrightarrow \varphi\left(x, x_{o} ; \sum_{j=1}^{2} \lambda_{j} g_{j}^{\prime}\left(x_{o} ; \theta_{j}\left(x, x_{o}\right)\right)\right) \\
+\tilde{\rho}^{2}\left\|\sigma\left(x, x_{o}\right)\right\|^{2}=-4\left(x_{1}^{2}+x_{2}^{2}\right) \leqq 0 .
\end{gathered}
$$

Therefore $(f, g)$ is pseudo-quasi $(\varphi, \widetilde{\alpha}, \widetilde{\rho}, \sigma)$ - $d_{I^{-}} V$-type I univex function at $x_{0}$.

However, for the above defined problem, if we take

(i) $x=(1,1)$

$$
\begin{aligned}
& b_{o}\left(x, x_{o}\right) \phi_{o}\left(f_{1}(x)-f_{1}\left(x_{o}\right)\right) \\
& <\varphi\left(x, x_{o} ; \alpha_{1}^{1}\left(x, x_{o}\right) f_{1}^{\prime}\left(x_{o} ; \eta_{1}\left(x, x_{o}\right)\right)\right) \\
& \quad+\rho_{1}^{1}\left\|\sigma\left(x, x_{o}\right)\right\|^{2} ;
\end{aligned}
$$

(ii) $x=(1,0)$,

$$
\begin{aligned}
& b_{o}\left(x, x_{o}\right) \phi_{o}\left(f_{2}(x)-f_{2}\left(x_{o}\right)\right) \\
& \quad<\varphi\left(x, x_{o} ; \alpha_{2}^{1}\left(x, x_{o}\right) f_{2}^{\prime}\left(x_{o} ; \eta_{2}\left(x, x_{o}\right)\right)\right) \\
& \quad+\rho_{2}^{1}\left\|\sigma\left(x, x_{o}\right)\right\|^{2} .
\end{aligned}
$$

So $(f, g)$ is not $(\varphi, \alpha, \rho, \sigma)-d_{I^{-}} V$-type I univex function at $x_{o}$.

\section{Sufficient Optimality Conditions}

In this section, we discuss some sufficient optimality conditions for a point to be an efficient solution of (MP) under newly defined class of $(\varphi, \alpha, \rho, \sigma)-d_{I^{-}} V$-type I univex functions.

Theorem 17. Suppose there exist a feasible solution $x_{0}$ of $(M P)$ and vector functions $\eta_{i}: X \times D \rightarrow R^{n}, i=\overline{1, m}, \theta_{j}: X \times D \rightarrow$ $R^{n}, j \in J\left(x_{o}\right)$, and scalars $\mu_{i}>0, i=\overline{1, m}$, and $\lambda_{j} \geqq 0, j \in$ $J\left(x_{0}\right)$ such that

(i) $\sum_{i=1}^{m} \mu_{i} f_{i}^{\prime}\left(x_{o} ; \eta_{i}\left(x, x_{o}\right)\right)+\sum_{j \in J\left(x_{o}\right)} \lambda_{j} g_{j}^{\prime}\left(x_{o} ; \theta_{j}\left(x, x_{o}\right)\right) \geqq$ $0, \forall x \in X$,

(ii) for any $u \in R, u<0 \Rightarrow \phi_{o}(u)<0$ and $u \geqq 0 \Rightarrow$ $\phi_{1}(u) \geqq 0, b_{o}\left(x, x_{o}\right)>0, b_{1}\left(x, x_{o}\right) \geqq 0$,

(iii) $\left(f, g_{J\left(x_{o}\right)}\right)$ is pseudo-quasi $(\varphi, \widetilde{\alpha}, \tilde{\rho}, \sigma)-d_{I}-V$-type I univex at $x_{o}$ with respect to $\left(\eta_{i}\right)_{i=1, m}$ and $\left(\theta_{j}\right)_{j \in J\left(x_{o}\right)}$, and

(iv) $\tilde{\rho}^{1}+\widetilde{\rho}^{2} \geqq 0$,

then $x_{o}$ is an efficient solution of (MP). 
Proof. Suppose that $x_{o}$ is not an efficient solution of (MP). Then there exists an $x \in X$ of (MP) such that $f(x) \leq f\left(x_{o}\right)$.

As $\mu_{i}>0, \tilde{\alpha}_{i}^{1}\left(x, x_{o}\right)>0, i=\overline{1, m}$, therefore

$$
\sum_{i=1}^{m} \mu_{i} \widetilde{\alpha}_{i}^{1}\left(x, x_{o}\right)\left(f_{i}(x)-f_{i}\left(x_{o}\right)\right)<0 .
$$

Also $g_{j}\left(x_{o}\right)=0, \lambda_{j} \geqq 0, \tilde{\alpha}_{j}^{2}\left(x, x_{o}\right)>0, j \in J\left(x_{o}\right)$ imply

$$
\sum_{j \in J\left(x_{o}\right)} \lambda_{j} \widetilde{\alpha}_{j}^{2}\left(x, x_{o}\right) g_{j}\left(x_{o}\right)=0 .
$$

Since hypothesis (ii) holds, therefore inequality (24) and equality (25) become

$$
\begin{gathered}
b_{o}\left(x, x_{o}\right) \phi_{o}\left(\sum_{i=1}^{m} \mu_{i} \widetilde{\alpha}_{i}^{1}\left(x, x_{o}\right)\left(f_{i}(x)-f_{i}\left(x_{o}\right)\right)\right)<0, \\
b_{1}\left(x, x_{o}\right) \phi_{1}\left(\sum_{j \in J\left(x_{o}\right)} \lambda_{j} \widetilde{\alpha}_{j}^{2}\left(x, x_{o}\right) g_{j}\left(x_{o}\right)\right) \geqq 0 .
\end{gathered}
$$

Using hypothesis (iii), we obtain

$$
\begin{gathered}
\varphi\left(x, x_{o} ; \sum_{i=1}^{m} \mu_{i} f_{i}^{\prime}\left(x_{o} ; \eta_{i}\left(x, x_{o}\right)\right)\right)<-\widetilde{\rho}^{1}\left\|\sigma\left(x, x_{o}\right)\right\|^{2}, \\
\varphi\left(x, x_{o} ; \sum_{j \in J\left(x_{o}\right)} \lambda_{j} g_{j}^{\prime}\left(x_{o} ; \theta_{j}\left(x, x_{o}\right)\right)\right) \leqq-\widetilde{\rho}^{2}\left\|\sigma\left(x, x_{o}\right)\right\|^{2} .
\end{gathered}
$$

The above inequalities along with subadditivity of $\varphi$ yield

$$
\begin{aligned}
& \varphi(x, x_{o} ; \sum_{i=1}^{m} \mu_{i} f_{i}^{\prime}\left(x_{o} ; \eta_{i}\left(x, x_{o}\right)\right) \\
&\left.+\sum_{j \in J\left(x_{o}\right)} \lambda_{j} g_{j}^{\prime}\left(x_{o} ; \theta_{j}\left(x, x_{o}\right)\right)\right) \\
&<-\left(\tilde{\rho}^{1}+\tilde{\rho}^{2}\right)\left\|\sigma\left(x, x_{o}\right)\right\|^{2} \leqq 0 \text { (using hypothesis (iv)). }
\end{aligned}
$$

But as hypothesis (i) holds and $\varphi\left(x, x_{0} ; 0\right)=0$ and $\varphi\left(x, x_{o} ; a\right)>0$ for $a>0$, therefore

$$
\begin{aligned}
\varphi(x, & x_{o} ; \sum_{i=1}^{m} \mu_{i} f_{i}^{\prime}\left(x_{o} ; \eta_{i}\left(x, x_{o}\right)\right) \\
& \left.+\sum_{j \in J\left(x_{o}\right)} \lambda_{j} g_{j}^{\prime}\left(x_{o} ; \theta_{j}\left(x, x_{o}\right)\right)\right) \geqq 0 .
\end{aligned}
$$

Thus we get a contradiction and hence the proof.

Theorem 18. Suppose there exist a feasible solution $x_{o}$ of $(M P)$ and vector functions $\eta_{i}: X \times D \rightarrow R^{n}, i=\overline{1, m}$, and $\theta_{j}$ : $X \times D \rightarrow R^{n}, j \in J\left(x_{o}\right)$, and scalars $\mu_{i} \geqq 0, i=\overline{1, m}$, and $\lambda_{j} \geqq 0, j \in J\left(x_{o}\right)$ satisfying (i) $\sum_{i=1}^{m} \mu_{i} f_{i}^{\prime}\left(x_{o} ; \eta_{i}\left(x, x_{o}\right)\right)+\sum_{j \in J\left(x_{o}\right)} \lambda_{j} g_{j}^{\prime}\left(x_{o} ; \theta_{j}\left(x, x_{o}\right)\right) \geqq$ 0 , for all $x \in X$,

(ii) for any $u \in R, u \leqq 0 \Rightarrow \phi_{o}(u) \leqq 0$ and $u \geqq 0 \Rightarrow$ $\phi_{1}(u) \geqq 0, b_{o}\left(x, x_{o}\right) \geqq 0, b_{1}\left(x, x_{o}\right) \geqq 0$,

(iii) $\left(f, g_{J\left(x_{0}\right)}\right)$ is strictly pseudo $(\varphi, \tilde{\alpha}, \tilde{\rho}, \sigma)-d_{I}-V$-type $I$ univex at $x_{o}$ with respect to $\left(\eta_{i}\right)_{i=1, m}$ and $\left(\theta_{j}\right)_{j \in J\left(x_{o}\right)}$, and

(iv) $\tilde{\rho}^{1}+\tilde{\rho}^{2} \geqq 0$,

then $x_{o}$ is an efficient solution of $(M P)$.

Proof. Suppose that $x_{o}$ is not an efficient solution of (MP). Then there exists $x \in X$ of (MP) such that $f(x) \leq f\left(x_{o}\right)$.

As $\mu_{i} \geqq 0, i=\overline{1, m}, \widetilde{\alpha}_{i}^{1}\left(x, x_{o}\right)>0, i=\overline{1, m}$, and $g_{j}\left(x_{o}\right)=0$, $\lambda_{j} \geqq 0, \widetilde{\alpha}_{j}^{2}\left(x, x_{o}\right)>0, j \in J\left(x_{o}\right)$, therefore

$$
\begin{gathered}
\sum_{i=1}^{m} \mu_{i} \tilde{\alpha}_{i}^{1}\left(x, x_{o}\right)\left(f_{i}(x)-f_{i}\left(x_{o}\right)\right) \leqq 0, \\
\sum_{j \in J\left(x_{o}\right)} \lambda_{j} \widetilde{\alpha}_{j}^{2}\left(x, x_{o}\right) g_{j}\left(x_{o}\right)=0 .
\end{gathered}
$$

Using hypothesis (ii), we obtain

$$
\begin{gathered}
b_{o}\left(x, x_{o}\right) \phi_{o}\left(\sum_{i=1}^{m} \mu_{i} \widetilde{\alpha}_{i}^{1}\left(x, x_{o}\right)\left(f_{i}(x)-f_{i}\left(x_{o}\right)\right)\right) \leqq 0, \\
b_{1}\left(x, x_{o}\right) \phi_{1}\left(\sum_{j \in J\left(x_{o}\right)} \lambda_{j} \widetilde{\alpha}_{j}^{2}\left(x, x_{o}\right) g_{j}\left(x_{o}\right)\right) \geqq 0 .
\end{gathered}
$$

Since $\left(f, g_{J\left(x_{o}\right)}\right)$ is strictly pseudo $(\varphi, \tilde{\alpha}, \tilde{\rho}, \sigma)-d_{I^{-}}-V$-type I univex at $x_{o}$, therefore, the above inequalities yield

$$
\begin{gathered}
\varphi\left(x, x_{o} ; \sum_{i=1}^{m} \mu_{i} f_{i}^{\prime}\left(x_{o} ; \eta_{i}\left(x, x_{o}\right)\right)\right)<-\widetilde{\rho}^{1}\left\|\sigma\left(x, x_{o}\right)\right\|^{2}, \\
\varphi\left(x, x_{o} ; \sum_{j \in J\left(x_{o}\right)} \lambda_{j} g_{j}^{\prime}\left(x_{o} ; \theta_{j}\left(x, x_{o}\right)\right)\right)<-\widetilde{\rho}^{2}\left\|\sigma\left(x, x_{o}\right)\right\|^{2} .
\end{gathered}
$$

Using subadditivity of $\varphi$ and hypothesis (iv), we get

$$
\begin{gathered}
\varphi\left(x, x_{o} ; \sum_{i=1}^{m} \mu_{i} f_{i}^{\prime}\left(x_{o} ; \eta_{i}\left(x, x_{o}\right)\right)\right. \\
\left.+\sum_{j \in J\left(x_{o}\right)} \lambda_{j} g_{j}^{\prime}\left(x_{o} ; \theta_{j}\left(x, x_{o}\right)\right)\right) \\
<-\left(\tilde{\rho}^{1}+\tilde{\rho}^{2}\right)\left\|\sigma\left(x, x_{o}\right)\right\|^{2} \leqq 0 .
\end{gathered}
$$

But hypothesis (i) and properties of $\varphi$ imply

$$
\begin{gathered}
\varphi\left(x, x_{o} ; \sum_{i=1}^{m} \mu_{i} f_{i}^{\prime}\left(x_{o} ; \eta_{i}\left(x, x_{o}\right)\right)\right. \\
\left.+\sum_{j \in J\left(x_{o}\right)} \lambda_{j} g_{j}^{\prime}\left(x_{o} ; \theta_{j}\left(x, x_{o}\right)\right)\right) \geqq 0,
\end{gathered}
$$


which leads to a contradiction. Hence $x_{o}$ is an efficient solution of (MP).

In order to illustrate the result obtained, we will give an example of a multiobjective optimization problem in which the efficient solution will be obtained by the application of Theorem 18.

Example 19. Let

$$
\begin{array}{ll}
\text { Minimize } & f(x)=\left(f_{1}(x), f_{2}(x)\right) \\
\text { subject to } & g(x)=\left(g_{1}(x), g_{2}(x)\right) \leqq 0,
\end{array}
$$

where $f: R^{2} \rightarrow R^{2}$ and $g: R^{2} \rightarrow R^{2}$ be defined as

$$
\begin{aligned}
& f_{1}\left(x_{1}, x_{2}\right)= \begin{cases}2+x_{2}^{2}+8\left|x_{1}\right| & \text { if } x_{1} \neq 0, x_{2} \neq 0 \\
0 & \text { if } x_{1}=0, x_{2}=0 \\
x_{1}^{2}+x_{2}^{2} & \text { else, }\end{cases} \\
& f_{2}\left(x_{1}, x_{2}\right)= \begin{cases}x_{1}^{2}+x_{2}^{2}-4\left|x_{1}\right| & \text { if } x_{1} \neq 0, x_{2} \neq 0 \\
0 & \text { if } x_{1}=0, x_{2}=0 \\
4+2 x_{1}^{2}+3 x_{2}^{4} & \text { else, }\end{cases} \\
& g_{1}\left(x_{1}, x_{2}\right)= \begin{cases}-x_{1}^{4}+1 & \text { if } x_{1} \neq 0, x_{2}=0 \\
0 & \text { if } x_{1}=0, x_{2}=0 \\
4 x_{1}-x_{1}^{2}-3 x_{2}^{2} & \text { else, }\end{cases} \\
& g_{2}\left(x_{1}, x_{2}\right)= \begin{cases}2 x_{1}-3 x_{1}^{4} & \text { if } x_{1} \neq 0, x_{2}=0 \\
0 & \text { if } x_{1}=0, x_{2}=0 \\
-x_{2}^{2}-x_{1}^{2}+2 & \text { else. }\end{cases}
\end{aligned}
$$

Let $\eta_{1}(x, y)=\left(1+x_{1}^{2}+y_{1}^{2}, 0\right), \eta_{2}(x, y)=\left(1+x_{1}^{2}, 2+2 x_{2}^{2}\right)$, $\theta_{1}(x, y)=\left(1+x_{1}^{2}, 2+x_{2}^{4}\right), \theta_{2}(x, y)=\left(2+2 x_{1}^{2}, 0\right), \varphi(x, y ; a)=$ $|a|\left(1+x_{1}^{2} y_{1}^{2}\right), b_{o}(x, y)=4, b_{1}(x, y)=4, \phi_{o}(t)=t / 2, \phi_{1}(t)=2 t$, and $\sigma(x, y)=\left(x_{1}+y_{1}, 1+y_{2}\right)$.

Also, let $\alpha_{1}^{1}(x, y)=4, \alpha_{2}^{1}(x, y)=2, \alpha_{1}^{2}(x, y)=2$, $\alpha_{2}^{2}(x, y)=1, \tilde{\rho}^{1}=4, \widetilde{\rho}^{2}=-4, \rho_{1}^{1}=0, \rho_{2}^{1}=4, \rho_{1}^{2}=$ $-2, \rho_{2}^{2}=-5, \mu_{1}=\mu_{2}=1 / 2, \lambda_{1}=1 / 2$, and $\lambda_{2}=0$ where $x=\left(x_{1}, x_{2}\right), y=\left(y_{1}, y_{2}\right), \widetilde{\alpha}_{i}^{1}(x, y)=1 / \alpha_{i}^{1}(x, y)$, and $\tilde{\alpha}_{j}^{2}(x, y)=1 / \alpha_{j}^{2}(x, y), i=1,2, j=1,2$.

The set $X$ of feasible solutions of problem is nonempty. Clearly, $f_{1}, f_{2}$ and $g_{1}, g_{2}$ are semidirectionally differentiable at $x_{o}=(0,0)$ with $f_{1}^{\prime}\left(x_{o} ; \eta_{1}\left(x, x_{0}\right)\right)=$ $0, f_{2}^{\prime}\left(x_{o} ; \eta_{2}\left(x, x_{o}\right)\right)=-4\left(1+x_{1}^{2}\right), g_{1}^{\prime}\left(x_{o} ; \theta_{1}\left(x, x_{o}\right)\right)=4\left(1+x_{1}^{2}\right)$, and $g_{2}^{\prime}\left(x_{o} ; \theta_{2}\left(x, x_{o}\right)\right)=4\left(1+x_{1}^{2}\right)$.

It is easy to see that for all $x \in X \backslash\left\{x_{0}\right\}$

$$
\begin{aligned}
& \varphi\left(x, x_{o} ; \sum_{i=1}^{2} \mu_{i} f_{i}^{\prime}\left(x_{o} ; \eta_{i}\left(x, x_{o}\right)\right)\right) \\
& \geqq-\tilde{\rho}^{1}\left\|\sigma\left(x, x_{o}\right)\right\|^{2} \\
& \Longrightarrow b_{o}\left(x, x_{o}\right) \phi_{o}\left(\sum_{i=1}^{2} \mu_{i} \tilde{\alpha}_{i}^{1}\left(x, x_{o}\right)\left(f_{i}(x)-f_{i}\left(x_{o}\right)\right)\right)>0,
\end{aligned}
$$

$$
\begin{aligned}
& b_{1}\left(x, x_{o}\right) \phi_{1}\left(\sum_{j=1}^{2} \lambda_{j} \widetilde{\alpha}_{j}^{2}\left(x, x_{o}\right) g_{j}\left(x_{o}\right)\right) \geqq 0 \\
& \Longrightarrow \varphi\left(x, x_{o} ; \sum_{j=1}^{2} \lambda_{j} g_{j}^{\prime}\left(x_{o} ; \theta_{j}\left(x, x_{o}\right)\right)\right) \\
& <-\tilde{\rho}^{2}\left\|\sigma\left(x, x_{o}\right)\right\|^{2} .
\end{aligned}
$$

Hence $\left(f, g_{J\left(x_{o}\right)}\right)$ is strictly pseudo $(\varphi, \widetilde{\alpha}, \widetilde{\rho}, \sigma)-d_{I}-V$-type I univex function at $x_{0}$.

Also, hypotheses (i), (ii), and (iv) of Theorem 18 are clearly satisfied and it follows that $x_{o}$ is an efficient solution of the above defined multiobjective optimization problem, whereas it will be impossible to apply for this purpose the sufficient optimality conditions given in Kharbanda et al. [18], Ahmad [17], Slimani and Radjef [12], Mishra and Noor [10], Mishra et al. [16], Antczak [9], Suneja and Srivastava [19], and Ye [8].

Theorem 20. Suppose there exist a feasible solution $x_{o}$ of $(M P)$ and vector functions $\eta_{i}: X \times D \rightarrow R^{n}, i=\overline{1, m}$, and $\theta_{j}$ : $X \times D \rightarrow R^{n}, j \in J\left(x_{o}\right)$, and scalars $\mu_{i} \geqq 0, i=\overline{1, m}$, and $\lambda_{j} \geqq 0, j \in J\left(x_{o}\right)$, satisfying

(i) $\sum_{i=1}^{m} \mu_{i} f_{i}^{\prime}\left(x_{o} ; \eta_{i}\left(x, x_{o}\right)\right)+\sum_{j \in J\left(x_{o}\right)} \lambda_{j} g_{j}^{\prime}\left(x_{o} ; \theta_{j}\left(x, x_{o}\right)\right) \geqq$ 0 , for all $x \in X$,

(ii) for any $u \in R, u \leqq 0 \Rightarrow \phi_{o}(u) \leqq 0$ and $u \geqq 0 \Rightarrow$ $\phi_{1}(u) \geqq 0, b_{o}\left(x, x_{o}\right) \geqq 0, b_{1}\left(x, x_{o}\right) \geqq 0$, and

(iii) $\tilde{\rho}^{1}+\widetilde{\rho}^{2} \geqq 0$.

Also, if either the fact that

(a) $\left(f, g_{J\left(x_{0}\right)}\right)$ is semistrictly quasi $(\varphi, \widetilde{\alpha}, \widetilde{\rho}, \sigma)-d_{I}-V$-type $I$ univex at $x_{o}$ with respect to $\left(\eta_{i}\right)_{i=1, m}$ and $\left(\theta_{j}\right)_{j \in J\left(x_{o}\right)}$, or

(b) $\left(f, g_{J\left(x_{o}\right)}\right)$ is quasistrictly pseudo $(\varphi, \widetilde{\alpha}, \tilde{\rho}, \sigma)-d_{I^{-}}-V$-type I univex at $x_{o}$ with respect to $\left(\eta_{i}\right)_{i=1, m}$ and $\left(\theta_{j}\right)_{j \in J\left(x_{o}\right)}$, or

(c) $\left(f, g_{J\left(x_{0}\right)}\right)$ is strictlypseudo-quasi $(\varphi, \tilde{\alpha}, \tilde{\rho}, \sigma)-d_{I}-V$-type I univex at $x_{o}$ with respect to $\left(\eta_{i}\right)_{i=\overline{1, m}}$ and $\left(\theta_{j}\right)_{j \in J\left(x_{o}\right)}$ holds,

then $x_{o}$ is an efficient solution of (MP).

Proof. If (a) or (c) holds, then proceeding as in previous theorem we get

$$
\begin{gathered}
\varphi\left(x, x_{o} ; \sum_{i=1}^{m} \mu_{i} f_{i}^{\prime}\left(x_{o} ; \eta_{i}\left(x, x_{o}\right)\right)\right)<-\tilde{\rho}^{1}\left\|\sigma\left(x, x_{o}\right)\right\|^{2}, \\
\varphi\left(x, x_{o} ; \sum_{j \in J\left(x_{o}\right)} \lambda_{j} g_{j}^{\prime}\left(x_{o} ; \theta_{j}\left(x, x_{o}\right)\right)\right) \leqq-\widetilde{\rho}^{2}\left\|\sigma\left(x, x_{o}\right)\right\|^{2} .
\end{gathered}
$$


And if (b) holds, we get

$$
\begin{gathered}
\varphi\left(x, x_{o} ; \sum_{i=1}^{m} \mu_{i} f_{i}^{\prime}\left(x_{o} ; \eta_{i}\left(x, x_{o}\right)\right)\right) \leqq-\tilde{\rho}^{1}\left\|\sigma\left(x, x_{o}\right)\right\|^{2}, \\
\varphi\left(x, x_{o} ; \sum_{j \in J\left(x_{o}\right)} \lambda_{j} g_{j}^{\prime}\left(x_{o} ; \theta_{j}\left(x, x_{o}\right)\right)\right)<-\widetilde{\rho}^{2}\left\|\sigma\left(x, x_{o}\right)\right\|^{2} .
\end{gathered}
$$

The remaining part of proof runs on the lines of the proof of Theorem 18.

Now, following Antczak [9] and Slimani and Radjef [12], we state the following necessary optimality conditions.

Theorem 21 (Karush-Kuhn-Tucker type necessary optimality conditions). If

(i) $x_{o}$ is a weakly efficient solution of $(M P)$,

(ii) $g_{j}$ is continuous at $x_{o}$ for $j \in \widetilde{J}\left(x_{o}\right)$,

(iii) there exist vector functions $\eta_{i}: X \times D \rightarrow R^{n}, i=\overline{1, m}$, and $\theta_{j}: X \times D \rightarrow R^{n}, j \in J\left(x_{o}\right)$, such that at $x_{o} \in D$ the following inequalities are satisfied with respect to $\eta: X \times D \rightarrow R^{n}:$

$$
\begin{array}{r}
f_{i}^{\prime}\left(x_{o} ; \eta\left(x, x_{o}\right)\right) \leqq f_{i}^{\prime}\left(x_{o} ; \eta_{i}\left(x, x_{o}\right)\right), \\
\forall x \in X, \forall i=\overline{1, m}, \\
g_{j}^{\prime}\left(x_{o} ; \eta\left(x, x_{o}\right)\right) \leqq g_{j}^{\prime}\left(x_{o} ; \theta_{j}\left(x, x_{o}\right)\right), \\
\forall x \in X, \forall j \in J\left(x_{o}\right),
\end{array}
$$

(iv) for all $i=\overline{1, m}$ and $j \in J\left(x_{o}\right), f_{i}$ and $g_{j}$ are semidirectionally differentiable at $x_{o}$ and the functions $f_{i}^{\prime}\left(x_{o} ; \eta_{i}\left(x, x_{o}\right)\right), i=\overline{1, m}$, and $g_{j}^{\prime}\left(x_{o} ; \theta_{j}\left(x, x_{o}\right)\right), j \in$ $J\left(x_{o}\right)$, are preinvex functions of $x$ on $X$,

(v) the function $g$ satisfies $d_{I}$-constraint qualification at $x_{o}$ with respect to $\left(\theta_{j}\right)_{j \in J\left(x_{o}\right)}$, then there exist $\mu \in R_{\geqslant}^{m}$ and $\lambda \in R_{\geqq}^{k}$ such that

$$
\sum_{i=1}^{m} \mu_{i} f_{i}^{\prime}\left(x_{o} ; \eta_{i}\left(x, x_{o}\right)\right)+\sum_{j=1}^{k} \lambda_{j} g_{j}^{\prime}\left(x_{o} ; \theta_{j}\left(x, x_{o}\right)\right) \geqq 0
$$

$\forall x \in X$,

$$
\lambda_{j} g_{j}\left(x_{o}\right)=0, \quad \forall j=\overline{1, k}
$$

\section{Mond-Weir Type Duality}

In this section, we consider Mond-Weir type dual of (MP) and establish weak, strong, converse, and strict converse duality theorems. Consider

$$
\begin{array}{cc}
\text { Max } & f(y) \\
\text { subject to } & \sum_{i=1}^{m} \mu_{i} f_{i}^{\prime}\left(y ; \eta_{i}(x, y)\right) \\
& +\sum_{j=1}^{k} \lambda_{j} g_{j}^{\prime}\left(y ; \theta_{j}(x, y)\right) \geqq 0, \\
\lambda_{j} g_{j}(y) \geqq 0, \quad j=\overline{1, k}, \quad \forall x \in X, &
\end{array}
$$

where $y \in D, \mu \in R_{\geqslant}^{m}, \lambda \in R_{\geqq}^{k}, \eta_{i}: X \times D \rightarrow R^{n}$, for all $i=\overline{1, m}$, and $\theta_{j}: X \times D \rightarrow R^{n}$, for all $j=\overline{1, k}$. Let $W$ be the set of feasible points of (MWD).

Theorem 22 (weak duality). Let $x$ and $\left(y, \mu, \lambda,\left(\eta_{i}\right)_{i=\overline{1, m}}\right.$, $\left.\left(\theta_{j}\right)_{j=1, k}\right)$ be the feasible solutions of (MP) and (MWD), respectively, with $\mu>0$. If

(i) $(f, g)$ is pseudo-quasi $(\varphi, \widetilde{\alpha}, \tilde{\rho}, \sigma)-d_{I^{-}} V$-type I univex at $y$ with respect to $\left(\eta_{i}\right)_{i=\overline{1, m}}$ and $\left(\theta_{j}\right)_{j=\overline{1, k}}$,

(ii) for any $u \in R, u<0 \Rightarrow \phi_{o}(u)<0$ and $u \geqq 0 \Rightarrow$ $\phi_{1}(u) \geqq 0, b_{o}(x, y)>0, b_{1}(x, y) \geqq 0$, and

(iii) $\tilde{\rho}^{1}+\tilde{\rho}^{2} \geqq 0$,

then

$$
f(x) \not \leq f(y) .
$$

Proof. Suppose to the contrary that

$$
f(x) \leqslant f(y) .
$$

As $\mu_{i}>0, \tilde{\alpha}_{i}^{1}(x, y)>0, i=\overline{1, m}$, we get

$$
\sum_{i=1}^{m} \mu_{i} \widetilde{\alpha}_{i}^{1}(x, y)\left(f_{i}(x)-f_{i}(y)\right)<0 .
$$

Since $\lambda_{j} g_{j}(y) \geqq 0, \widetilde{\alpha}_{j}^{2}(x, y)>0, j=\overline{1, k}$, therefore

$$
\sum_{j=1}^{k} \lambda_{j} \widetilde{\alpha}_{j}^{2}(x, y) g_{j}(y) \geqq 0 .
$$

Using hypothesis (ii), we get

$$
b_{o}(x, y) \phi_{o}\left(\sum_{i=1}^{m} \mu_{i} \tilde{\alpha}_{i}^{1}(x, y)\left(f_{i}(x)-f_{i}(y)\right)\right)<0,
$$

$$
b_{1}(x, y) \phi_{1}\left(\sum_{j=1}^{k} \lambda_{j} \widetilde{\alpha}_{j}^{2}(x, y) g_{j}(y)\right) \geqq 0 .
$$


Since hypothesis (i) holds, therefore the above inequalities yield

$$
\begin{gathered}
\varphi\left(x, y ; \sum_{i=1}^{m} \mu_{i} f_{i}^{\prime}\left(y ; \eta_{i}(x, y)\right)\right)<-\tilde{\rho}^{1}\|\sigma(x, y)\|^{2}, \\
\varphi\left(x, y ; \sum_{j=1}^{k} \lambda_{j} g_{j}^{\prime}\left(y ; \theta_{j}(x, y)\right)\right) \leqq-\widetilde{\rho}^{2}\|\sigma(x, y)\|^{2} .
\end{gathered}
$$

Using subadditivity of $\varphi$ and hypothesis (iii), we get

$$
\begin{gathered}
\varphi\left(x, y ; \sum_{i=1}^{m} \mu_{i} f_{i}^{\prime}\left(y ; \eta_{i}(x, y)\right)+\sum_{j=1}^{k} \lambda_{j} g_{j}^{\prime}\left(y ; \theta_{j}(x, y)\right)\right) \\
<-\left(\tilde{\rho}^{1}+\tilde{\rho}^{2}\right)\|\sigma(x, y)\|^{2} \leqq 0 .
\end{gathered}
$$

However, the feasible condition (42) and properties of $\varphi$ imply

$$
\varphi\left(x, y ; \sum_{i=1}^{m} \mu_{i} f_{i}^{\prime}\left(y ; \eta_{i}(x, y)\right)+\sum_{j=1}^{k} \lambda_{j} g_{j}^{\prime}\left(y ; \theta_{j}(x, y)\right)\right) \geqq 0 .
$$

Thus we get a contradiction and hence the proof.

The proof of the following theorems runs on the lines of the proof of Theorem 22 .

Theorem 23 (weak duality). Let $x$ and $\left(y, \mu, \lambda,\left(\eta_{i}\right)_{i=\overline{1, m}}\right.$, $\left.\left(\theta_{j}\right)_{j=1, k}\right)$ be the feasible solutions of (MP) and (MWD), respectively. If

(i) $(f, g)$ is pseudo-quasi $(\varphi, \tilde{\alpha}, \tilde{\rho}, \sigma)-d_{I}-V$-type I univex at $y$ with respect to $\left(\eta_{i}\right)_{i=\overline{1, m}}$ and $\left(\theta_{j}\right)_{j=\overline{1, k}}$,

(ii) for any $u \in R, u<0 \Rightarrow \phi_{o}(u)<0$ and $u \geqq 0 \Rightarrow$ $\phi_{1}(u) \geqq 0, b_{o}(x, y)>0, b_{1}(x, y) \geqq 0$, and

(iii) $\tilde{\rho}^{1}+\tilde{\rho}^{2} \geqq 0$,

then

$$
f(x) \nless f(y) .
$$

Theorem 24 (weak duality). Let $x$ and $\left(y, \mu, \lambda,\left(\eta_{i}\right)_{i=\overline{1, m}}\right.$, $\left.\left(\theta_{j}\right)_{j=1, k}\right)$ be the feasible solutions of (MP) and (MWD), respectively. If

(i) $(f, g)$ is quasistrictly pseudo $(\varphi, \tilde{\alpha}, \tilde{\rho}, \sigma)-d_{I}-V$-type $I$ univex at $y$ with respect to $\left(\eta_{i}\right)_{i=\overline{1, m}}$ and $\left(\theta_{j}\right)_{j=\overline{1, k}}$,

(ii) for any $u \in R, u \leqq 0 \Rightarrow \phi_{o}(u) \leqq 0$ and $u \geqq 0 \Rightarrow$ $\phi_{1}(u) \geqq 0, b_{o}(x, y) \geqq 0, b_{1}(x, y) \geqq 0$, and

(iii) $\tilde{\rho}^{1}+\tilde{\rho}^{2} \geqq 0$,

then

$$
f(x) \not \leq f(y) .
$$

Theorem 25 (strong duality). Let $x_{0}$ be a weakly efficient solution of $(M P)$ and $g_{j}$ is continuous at $x_{o}$ for $j \in \widetilde{J}\left(x_{o}\right)$. Also, the vector functions $\eta_{i}, i=\overline{1, m}$ and $\theta_{j}, j \in J\left(x_{o}\right)$ exist for which

$$
\begin{array}{r}
f_{i}^{\prime}\left(x_{o} ; \eta\left(x, x_{o}\right)\right) \leqq f_{i}^{\prime}\left(x_{o} ; \eta_{i}\left(x, x_{o}\right)\right), \\
\forall x \in X, \forall i=\overline{1, m}, \\
g_{j}^{\prime}\left(x_{o} ; \eta\left(x, x_{o}\right)\right) \leqq g_{j}^{\prime}\left(x_{o} ; \theta_{j}\left(x, x_{o}\right)\right), \\
\forall x \in X, \forall j \in J\left(x_{o}\right)
\end{array}
$$

at $x_{o}$ and $f_{i}, g_{j}$ are semidirectionally differentiable at $x_{o}$ with $f_{i}^{\prime}\left(x_{o} ; \eta_{i}\left(x, x_{o}\right)\right)$ and $g_{j}^{\prime}\left(x_{o} ; \theta_{j}\left(x, x_{o}\right)\right)$ as preinvex functions on $X$. Also if $g$ satisfies $d_{I}$-constraint qualification at $x_{o}$, then $\exists \mu \in R_{\geq}^{m}$ and $\lambda \in R_{\geqq}^{k}$ such that $\left(x_{o}, \mu, \lambda,\left(\eta_{i}\right)_{i=\overline{1, m}},\left(\theta_{j}\right)_{j=\overline{1, k}}\right)$ is feasible for (MWD) and the objective function values of (MP) and (MWD) are equal. Moreover, if any weak duality holds, then $\left(x_{o}, \mu, \lambda,\left(\eta_{i}\right)_{i=\overline{1, m}},\left(\theta_{j}\right)_{j=\overline{1, k}}\right)$ is a weakly efficient solution of (MWD).

Proof. Since $x_{o}$ is a weakly efficient solution of (MP), therefore, by Theorem 21 , there exist $\mu \in R_{\geqslant}^{m}$ and $\lambda \in R_{\geqq}^{k}$ such that

$$
\begin{gathered}
\sum_{i=1}^{m} \mu_{i} f_{i}^{\prime}\left(x_{o} ; \eta_{i}\left(x, x_{o}\right)\right)+\sum_{j=1}^{k} \lambda_{j} g_{j}^{\prime}\left(x_{o} ; \theta_{j}\left(x, x_{o}\right)\right) \geqq 0, \\
\lambda_{j} g_{j}\left(x_{o}\right)=0, \quad \forall j=\overline{1, k} . X,
\end{gathered}
$$

It follows that $\left(x_{o}, \mu, \lambda,\left(\eta_{i}\right)_{i=\overline{1, m}},\left(\theta_{j}\right)_{j=\overline{1, k}}\right) \in W$ and therefore feasible for (MWD). Clearly, objective function values of (MP) and (MWD) are equal at these points.

Suppose $\left(x_{o}, \mu, \lambda,\left(\eta_{i}\right)_{i=\overline{1, m}},\left(\theta_{j}\right)_{j=\overline{1, k}}\right)$ is not a weakly efficient solution for (MWD). Then $\exists\left(\tilde{y}, \tilde{\mu}, \tilde{\lambda},\left(\widetilde{\eta}_{i}\right)_{i=\overline{1, m}},\left(\widetilde{\theta}_{j}\right)_{j=\overline{1, k}}\right)$ $\in W$ such that $f\left(x_{o}\right)<f(\tilde{y})$ which contradicts weak duality theorems. Therefore $\left(x_{o}, \mu, \lambda,\left(\eta_{i}\right)_{i=\overline{1, m}},\left(\theta_{j}\right)_{j=\overline{1, k}}\right)$ is a weakly efficient solution of (MWD).

Theorem 26 (converse duality). Let $\left(y_{o}, \mu, \lambda,\left(\eta_{i}\right)_{i=\overline{1, m}}\right.$, $\left.\left(\theta_{j}\right)_{j=1, k}\right)$ be a feasible solution of (MWD). Suppose hypotheses of Theorem 24 hold at $y_{o}$, then $y_{o}$ is an efficient solution of (MP).

Proof. Suppose that $y_{o}$ is not an efficient solution of (MP). Then $\exists x_{o} \in X$ such that

$$
f\left(x_{o}\right) \leqslant f\left(y_{o}\right) .
$$

Hence, by Theorem 24 (weak duality), we obtain a contradiction. Therefore $y_{o}$ is an efficient solution of (MP).

Theorem 27 (strict converse duality). Let $x_{o}$ and $\left(y_{o}, \mu, \lambda\right.$, $\left.\left(\eta_{i}\right)_{i=\overline{1, m}},\left(\theta_{j}\right)_{j=\overline{1, k}}\right)$ be the feasible solutions of (MP) and (MWD), respectively. If

(i) $f\left(x_{o}\right) \leqq f\left(y_{o}\right)$, 
(ii) $(f, g)$ is semistrictly quasi $(\varphi, \tilde{\alpha}, \tilde{\rho}, \sigma)-d_{I}-V$-type I univex at $y_{o}$ with respect to $\left(\eta_{i}\right)_{i=\overline{1, m}}$ and $\left(\theta_{j}\right)_{j=\overline{1, k}}$,

(iii) for any $u \in R, u \leqq 0 \Rightarrow \phi_{o}(u) \leqq 0$ and $u \geqq 0 \Rightarrow$ $\phi_{1}(u) \geqq 0, b_{o}\left(x_{o}, y_{o}\right) \geqq 0, b_{1}\left(x_{o}, y_{o}\right) \geqq 0$, and

(iv) $\tilde{\rho}^{1}+\tilde{\rho}^{2} \geqq 0$,

then $x_{o}=y_{o}$.

Proof. Suppose $x_{o} \neq y_{o}$.

Since $\mu \in R_{\geq}^{m}, \widetilde{\alpha}_{i}^{1}\left(x_{o}, y_{o}\right)>0, i=\overline{1, m}$, and hypothesis (i) holds, therefore

$$
\sum_{i=1}^{m} \mu_{i} \tilde{\alpha}_{i}^{1}\left(x_{o}, y_{o}\right) f_{i}\left(x_{o}\right) \leqq \sum_{i=1}^{m} \mu_{i} \tilde{\alpha}_{i}^{1}\left(x_{o}, y_{o}\right) f_{i}\left(y_{o}\right) .
$$

As $\tilde{\alpha}_{j}^{2}\left(x_{o}, y_{o}\right)>0, j=\overline{1, k}$, and $y_{o}$ is feasible solution of (MWD), therefore

$$
\sum_{j=1}^{k} \lambda_{j} \widetilde{\alpha}_{j}^{2}\left(x_{o}, y_{o}\right) g_{j}\left(y_{o}\right) \geqq 0 .
$$

Using hypothesis (iii), we obtain

$$
\begin{gathered}
b_{o}\left(x_{o}, y_{o}\right) \phi_{o}\left(\sum_{i=i}^{m} \mu_{i} \tilde{\alpha}_{i}^{1}\left(x_{o}, y_{o}\right)\left(f_{i}\left(x_{o}\right)-f_{i}\left(y_{o}\right)\right)\right) \leqq 0, \\
b_{1}\left(x_{o}, y_{o}\right) \phi_{1}\left(\sum_{j=1}^{k} \lambda_{j} \widetilde{\alpha}_{j}^{2}\left(x_{o}, y_{o}\right) g_{j}\left(y_{o}\right)\right) \geqq 0 .
\end{gathered}
$$

Applying hypothesis (ii) to the above inequalities, we get

$$
\begin{gathered}
\varphi\left(x_{o}, y_{o} ; \sum_{i=1}^{m} \mu_{i} f_{i}^{\prime}\left(y_{o} ; \eta_{i}\left(x_{o}, y_{o}\right)\right)\right)<-\tilde{\rho}^{1}\left\|\sigma\left(x_{o}, y_{o}\right)\right\|^{2}, \\
\varphi\left(x_{o}, y_{o} ; \sum_{j=1}^{k} \lambda_{j} g_{j}^{\prime}\left(y_{o} ; \theta_{j}\left(x_{o}, y_{o}\right)\right)\right) \leqq-\widetilde{\rho}^{2}\left\|\sigma\left(x_{o}, y_{o}\right)\right\|^{2} .
\end{gathered}
$$

Using subadditivity of $\varphi$ and hypothesis (iv), we get

$$
\begin{gathered}
\varphi\left(x_{o}, y_{o} ; \sum_{i=1}^{m} \mu_{i} f_{i}^{\prime}\left(y_{o} ; \eta_{i}\left(x_{o}, y_{o}\right)\right)\right. \\
\left.+\sum_{j=1}^{k} \lambda_{j} g_{j}^{\prime}\left(y_{o} ; \theta_{j}\left(x_{o}, y_{o}\right)\right)\right)<0
\end{gathered}
$$

which is a contradiction as feasible condition (42) holds and $\varphi\left(x_{o}, y_{o} ; 0\right)=0$ and $\varphi\left(x_{o}, y_{o} ; a\right)>0$ for $a>0$ yield

$$
\begin{gathered}
\varphi\left(x_{o}, y_{o} ; \sum_{i=1}^{m} \mu_{i} f_{i}^{\prime}\left(y_{o} ; \eta_{i}\left(x_{o}, y_{o}\right)\right)\right. \\
\left.+\sum_{j=1}^{k} \lambda_{j} g_{j}^{\prime}\left(y_{o} ; \theta_{j}\left(x_{o}, y_{o}\right)\right)\right) \geqq 0 .
\end{gathered}
$$

Hence $x_{o}=y_{o}$.

\section{Conflict of Interests}

The authors declare that there is no conflict of interests regarding the publication of this paper.

\section{References}

[1] M. A. Hanson, "On sufficiency of the Kuhn-Tucker conditions," Journal of Mathematical Analysis and Applications, vol. 80, no. 2, pp. 545-550, 1981.

[2] J.-P. Vial, "Strong and weak convexity of sets and functions," Mathematics of Operations Research, vol. 8, no. 2, pp. 231-259, 1983.

[3] M. A. Hanson and B. Mond, "Necessary and sufficient conditions in constrained optimization," Mathematical Programming, vol. 37, no. 1, pp. 51-58, 1987.

[4] V. Jeyakumar and B. Mond, "On generalised convex mathematical programming," Australian Mathematical Society B, vol. 34, no. 1, pp. 43-53, 1992.

[5] M. A. Hanson, R. Pini, and C. Singh, "Multiobjective programming under generalized type I invexity," Journal of Mathematical Analysis and Applications, vol. 261, no. 2, pp. 562-577, 2001.

[6] Z. A. Liang, H. X. Huang, and P. M. Pardalos, "Optimality conditions and duality for a class of nonlinear fractional programming problems," Journal of Optimization Theory and Applications, vol. 110, no. 3, pp. 611-619, 2001.

[7] T. R. Gulati, I. Ahmad, and D. Agarwal, "Sufficiency and duality in multiobjective programming under generalized type I functions," Journal of Optimization Theory and Applications, vol. 135, no. 3, pp. 411-427, 2007.

[8] Y. L. Ye, "d-invexity and optimality conditions," Journal of Mathematical Analysis and Applications, vol. 162, no. 1, pp. 242249, 1991.

[9] T. Antczak, "Multiobjective programming under $d$-invexity," European Journal of Operational Research, vol. 137, no. 1, pp. 2836, 2002.

[10] S. K. Mishra and M. A. Noor, "Some nondifferentiable multiobjective programming problems," Journal of Mathematical Analysis and Applications, vol. 316, no. 2, pp. 472-482, 2006.

[11] C. Nahak and R. N. Mohapatra, " $d-\rho-\eta-\theta$-invexity in multiobjective optimization," Nonlinear Analysis: Theory, Methods and Applications, vol. 70, no. 6, pp. 2288-2296, 2009.

[12] H. Slimani and M. S. Radjef, "Nondifferentiable multiobjective programming under generalized $d_{I}$-invexity," European Journal of Operational Research, vol. 202, no. 1, pp. 32-41, 2010.

[13] C. R. Bector, S. K. Suneja, and S. Gupta, "Univex functions and univex nonlinear programming," in Proceeding of the Administrative Sciences Association of Canada, pp. 115-124, 1992.

[14] N. G. Rueda, M. A. Hanson, and C. Singh, "Optimality and duality with generalized convexity," Journal of Optimization Theory and Applications, vol. 86, no. 2, pp. 491-500, 1995.

[15] S. K. Mishra, "On multiple-objective optimization with generalized univexity," Journal of Mathematical Analysis and Applications, vol. 224, no. 1, pp. 131-148, 1998.

[16] S. K. Mishra, S. Y. Wang, and K. K. Lai, "Nondifferentiable multiobjective programming under generalized $d$-univexity," European Journal of Operational Research, vol. 160, no. 1, pp. 218-226, 2005.

[17] I. Ahmad, "Efficiency and duality in nondifferentiable multiobjective programming involving directional derivative," Applied Mathematics, vol. 2, no. 4, pp. 452-460, 2011. 
[18] P. Kharbanda, D. Agarwal, and D. Sinha, "Nonsmooth multiobjective optimization involving generalized univex functions," Opsearch, 2013.

[19] S. K. Suneja and M. K. Srivastava, "Optimality and duality in nondifferentiable multiobjective optimization involving $d$-type I and related functions," Journal of Mathematical Analysis and Applications, vol. 206, no. 2, pp. 465-479, 1997.

[20] T. Weir and B. Mond, "Pre-invex functions in multiple objective optimization," Journal of Mathematical Analysis and Applications, vol. 136, no. 1, pp. 29-38, 1988.

[21] T. Weir and V. Jeyakumar, "A class of nonconvex functions and mathematical programming," Bulletin of the Australian Mathematical Society, vol. 38, no. 2, pp. 177-189, 1988.

[22] S. Mititelu, “Invex sets," Studii și Cercetări Matematice, vol. 46, no. 5, pp. 529-532, 1994.

[23] F. H. Clarke, Optimization and Nonsmooth Analysis, John Wiley \& Sons, New York, NY, USA, 1983. 


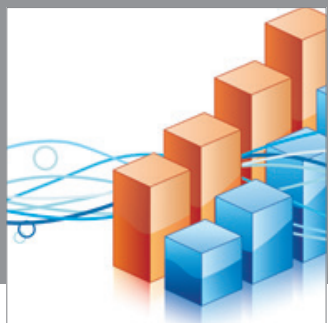

Advances in

Operations Research

mansans

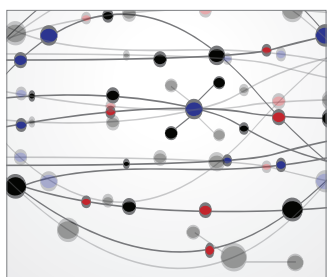

The Scientific World Journal
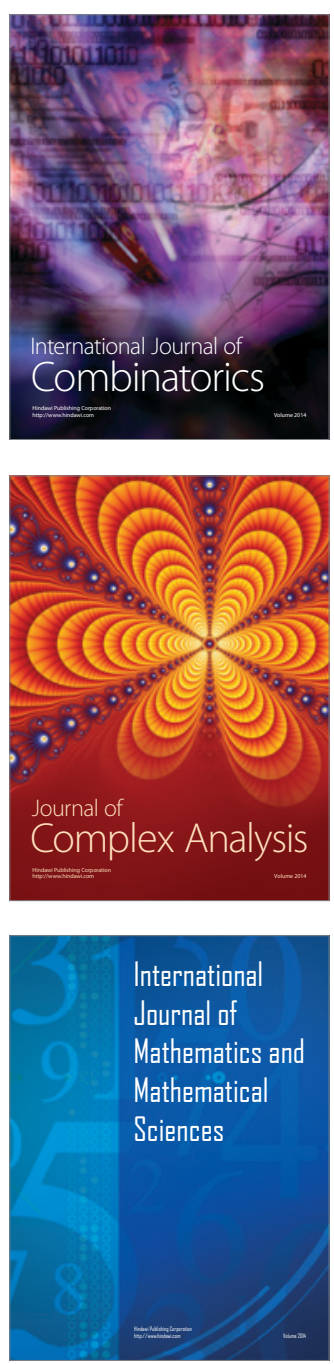
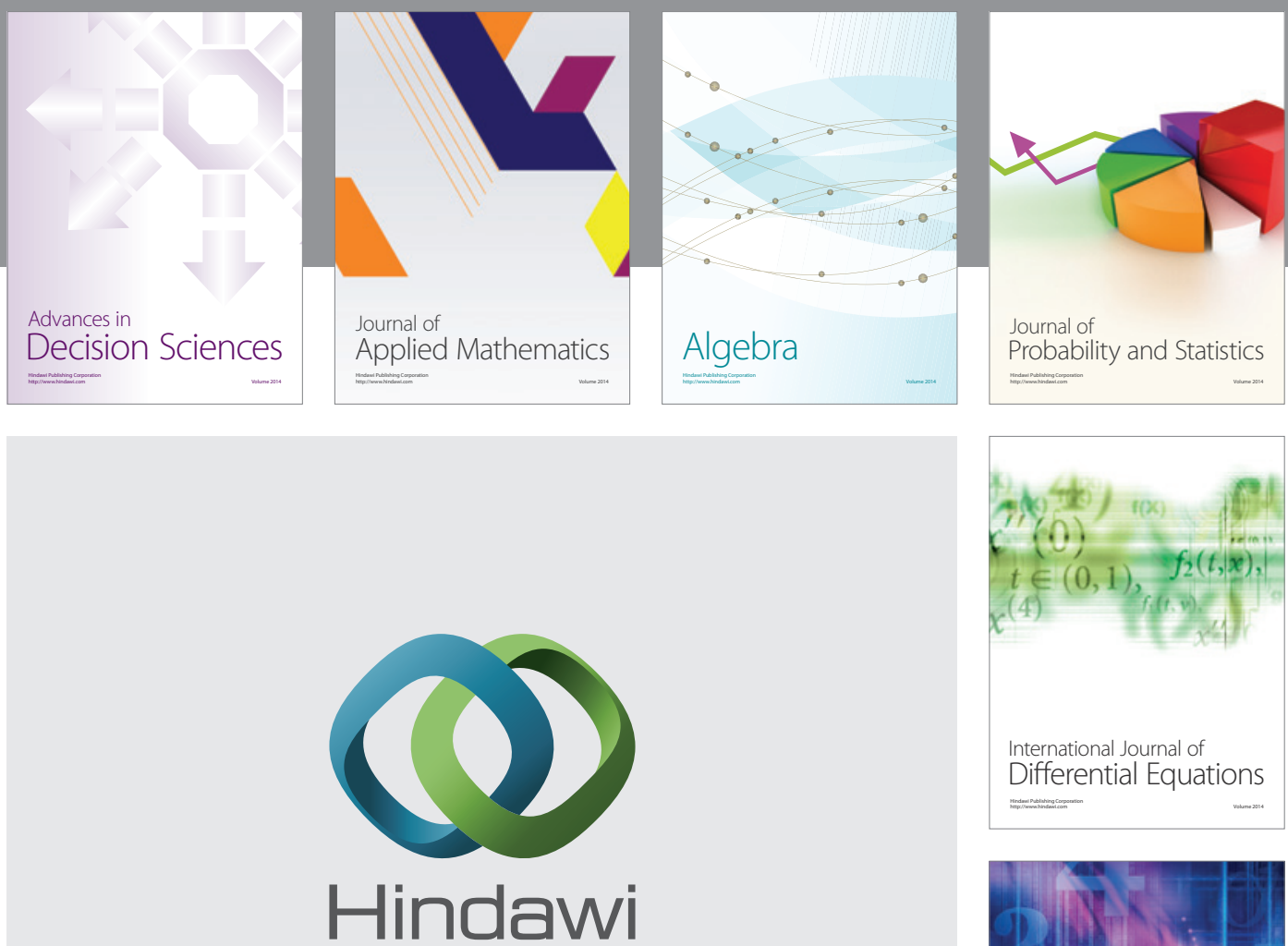

Submit your manuscripts at http://www.hindawi.com
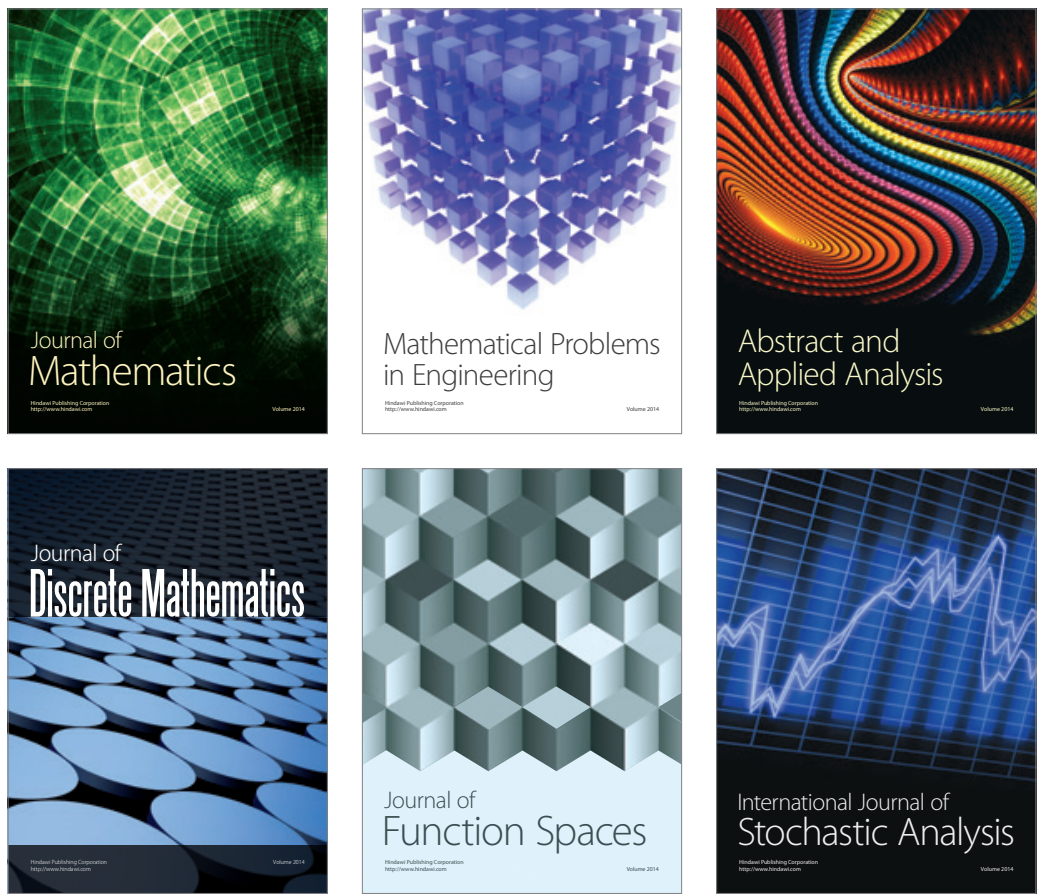

Journal of

Function Spaces

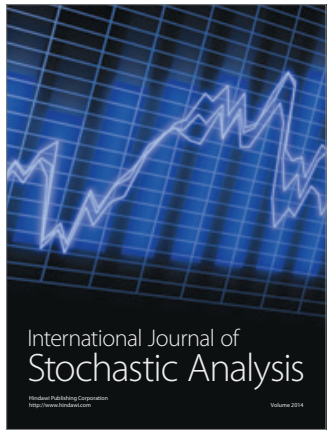

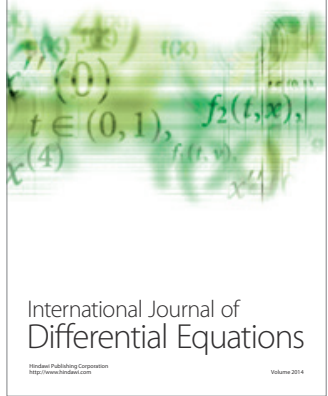
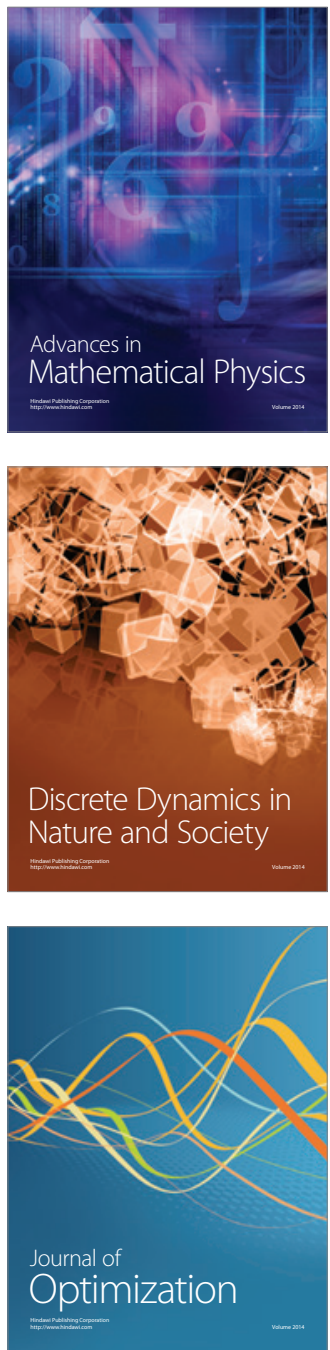